\title{
Antitumor activity of total flavonoids from Tetrastigma hemsleyanum Diels et Gilg is associated with the inhibition of regulatory $T$ cells in mice
}

\author{
This article was published in the following Dove Press journal: \\ OncoTargets and Therapy \\ 9 June 2014 \\ Number of times this article has been viewed
}

\author{
Zhengquan Feng ${ }^{1,2}$ \\ Wanrong $\mathrm{Hao}^{3}$ \\ Xiaoyang $\operatorname{Lin}^{3}$ \\ Daping Fan ${ }^{4}$ \\ Juhua Zhou ${ }^{5}$
}

'Department of Oncology, Tongde Hospital of Zhejiang Province,

Hangzhou, Zhejiang,

People's Republic of China;

'Department of Oncology,

Guang An Men Hospital,

China Academy of Chinese

Medical Sciences, Beijing,

People's Republic of China;

${ }^{3}$ Department of Chinese Medicine,

Zhejiang Chinese Medical

University, Hangzhou,

Zhejiang, People's Republic

of China; ${ }^{4}$ Department of Cell

Biology and Anatomy, School of

Medicine, University of South

Carolina, Columbia, SC, USA;

${ }^{5}$ Institute for Tumor Immunology,

Ludong University College of Life

Sciences, Yantai, Shandong, People's

Republic of China
Correspondence: Juhua Zhou

Institute for Tumor Immunology,

Ludong University School of Life

Sciences, 186 Hongqi Middle Road,

Yantai, Shandong 264025,

People's Republic of China

Tel +86 I83 00501903

Fax +860535 6642910

Email juhua.zhou@gmail.com
Objective: To determine the antitumor activity of Radix tetrastigmae flavonoids and their inhibitory effect on regulatory $\mathrm{T}$ cells (Tregs) in mice.

Materials and methods: Total flavonoids were isolated from Radix tetrastigmae, the root of Tetrastigma hemsleyanum Diels et Gilg, and administered to C57BL/6 mice by oral gavage after inoculation with Lewis lung carcinoma (LLC) cells. The effects of total flavonoids on tumor growth in vivo were examined. Flow cytometry was used to study the effects on Tregs, and enzyme-linked immunosorbent assay was used to analyze the changes in the serum levels of transforming growth factor $\beta$, prostaglandin E2, and cyclooxygenase 2 after tumor inoculation and flavonoid administration.

Results: Total flavonoids from $T$. hemsleyanum Diels et Gilg significantly inhibited tumor growth in C57BL/6 mice inoculated with LLCs. These flavonoids dramatically suppressed regulatory T-cell development in tumor-bearing mice. Further studies revealed that total flavonoids significantly decreased the serum levels of transforming growth factor $\beta$, prostaglandin E2, and cyclooxygenase 2 in tumor-bearing mice, which may be responsible for the inhibition of Tregs.

Conclusion: The antitumor activity of total flavonoids from T. hemsleyanum Diels et Gilg is associated with the inhibition of Tregs in a mouse tumor model. Total flavonoids from T. hemsleyanum Diels et Gilg may be used as antitumor agents in cancer prevention and treatment.

Keywords: flavonoids, radix tetrastigmae, regulatory T cells, tumor growth, TGF- $\beta 1$

\section{Introduction}

It has been recognized that flavonoids have antitumor activity at several stages in cancer development and progression, and thus can potentially be used as antitumor agents. ${ }^{1}$ However, the mechanisms of flavonoid-mediated antitumor functions are not fully understood. It has been reported that flavonoids from plants such as Macrothelypteris viridifrons and Vitex rotundifolia have strong antiproliferative effects on tumor cells. ${ }^{2,3}$ They may induce tumor necrosis factor-related apoptosisinducing ligand-mediated apoptosis in cancer cells. ${ }^{4}$ Other studies showed that flavonoids could inhibit the activation of nuclear factor kappa-light chain enhancer of activated B cells and suppress the activities of epidermal growth factor and kinase insert domain receptor tyrosine kinases, and thus induce apoptosis in tumor cells. ${ }^{5}$ Flavonoids also inhibit the release of angiogenic factors, particularly vascular endothelial growth factor, from human breast cancer cells, and thus suppress tumor 
development and progression. ${ }^{6}$ Furthermore, flavonoids inhibit the expression of vascular cell adhesion molecule 1 in human umbilical vein endothelial cells and murine pulmonary endothelial cells ${ }^{7}$ and attenuate the expression of E-selectin and intercellular adhesion molecule 1 in human endothelial cells, ${ }^{8}$ suggesting flavonoids may impair the interactions between tumor cells and endothelial cells, and thus inhibit melanoma lung metastasis in mice. Therefore, flavonoids may be used as effective preventive and therapeutic agents, although the mechanisms of their actions are unclear. ${ }^{9-11}$

It has been documented that regulatory $\mathrm{T}$ cells (Tregs) play an important inhibitory role in the regulation of immune responses. ${ }^{12,13}$ Tregs express CD4, CD25, and Foxp3. ${ }^{14}$ They are often associated with solid tumors in both cancer patients and animal models. Increased numbers of Tregs have been observed in breast, colorectal, and ovarian cancers. ${ }^{15}$ Inhibitory activities of Tregs on other CD4 T cells and cytotoxic CD8 T cells depend on cell-cell contact and secretion of inhibitory cytokines such as interleukin 10 and transforming growth factor beta (TGF- $\beta$ ). ${ }^{16}$ Tregs contribute to cancer development and are responsible for the failure of current therapies mediated by antitumor responses. ${ }^{17}$ Several factors such as interleukin 2 and TGF- $\beta$ are essential for Treg development and homeostasis. ${ }^{18,19}$ Chemotherapeutic agents such as cyclophosphamide and paclitaxel inhibit Tregs, which are partially responsible for their antitumor activities. ${ }^{20-22}$ However, little is known about the role of flavonoids in the regulation of Treg development and antitumor immunity.

Our previous studies indicated that total flavonoids isolated from Radix tetrastigmae (the root of Tetrastigma hemsleyanum Diels et Gilg) could inhibit the cell growth of SGC-7901 human gastric cancer cell line in vitro. ${ }^{23}$ In the current study, we found that total flavonoids from T. hemsleyanum Diels et Gilg could significantly inhibit the tumor growth of the Lewis lung carcinoma (LLC) cell line and Tregs in mice. The results suggest that the antitumor activity of total flavonoids from T. hemsleyanum Diels et Gilg is associated with the inhibition of Tregs in vivo in a mouse tumor model.

\section{Materials and methods}

\section{Materials and total flavonoid isolation}

Radix Tetrastigmae, the root of $T$. hemsleyanum Diels et Gilg, was purchased from the pharmaceutical store in the Zhejiang Chinese Medical University-affiliated First Hospital, Hangzhou, People's Republic of China.
Total flavonoids were extracted from Radix Tetrastigmae according to the method reported by Liu et al. ${ }^{24}$ Briefly, the powdered root (100 g) of T. hemsleyanum Diels et Gilg was extracted twice with $600 \mathrm{~mL}$ of $60 \%$ ethanol in a boiling water bath for 90 minutes and then filtered. The filtrate was concentrated under vacuum at $45^{\circ} \mathrm{C}$, freeze-dried, and stored at $4^{\circ} \mathrm{C}$. Approximately $2 \mathrm{~g}$ of the total flavonoid extract was obtained. The quality of the total flavonoid extract was determined by colorimetric HCl-Mg and aluminum chloride reactions, followed by high-performance liquid chromatography. ${ }^{24,25}$

\section{Mice and tumor inoculation}

Healthy wild-type C57BL/6 (B6) mice without immunosuppression and other immunological disorders were purchased from the Shanghai Laboratory Animal Center, Chinese Academy of Sciences, Shanghai, People's Republic of China, and housed in the Laboratory Animal Center at Zhejiang Chinese Medical University, Hangzhou, People's Republic of China. The animal protocol was approved by the Institutional Animal Use Committee of Zhejiang Chinese Medical University. All mice were maintained under pathogen-free conditions.

The LLC cell line was purchased from the Shanghai Institute of Cell Biology, Chinese Academy of Sciences, Shanghai, People's Republic of China, and maintained in RPMI 1640 medium with 5\% fetal calf serum in a humidified $5 \% \mathrm{CO}_{2}$ incubator. After washing twice, LLC cells were suspended in phosphate-buffered saline (PBS). LLC cells $\left(2 \times 10^{6}\right)$ in $200 \mu \mathrm{L}$ PBS were subcutaneously injected in the right axillary fossa of each 2-month-old mouse. The same volume of PBS was subcutaneously injected in the right axillary fossa of each 2-month-old mouse as control.

\section{Total flavonoid administration}

Starting on the second day after tumor inoculation, the total flavonoids extracted from Radix Tetrastigmae were dissolved in $0.9 \%$ saline solution and administered in mice by oral gavage once daily at doses of $0,5,10$, or $15 \mathrm{mg} / \mathrm{kg}$ mouse body weight. Each group consisted of 10 mice. Changes of mouse appearance, mouse body weight, and tumor size were examined and recorded daily. Tumor volumes were calculated on the basis of the measurement of tumor sizes according to the previously published method of Euhus et al. ${ }^{26}$ At 14 days after tumor inoculation and total flavonoid administration, mice were killed for tumor weight determination, peripheral blood collection, and splenocyte preparation. 


\section{Examination of transmission electron microscopy}

Tumor cells were separated from mice at day 14 after tumor cell inoculation and flavonoid administration and were fixed in $4 \%$ glutaraldehyde for 2 hours. After washing with PBS, the cells were fixed in $1 \% \mathrm{OsO}_{4}$ for 1 hour. Then, the cell samples were dehydrated by graded alcohol, embedded with Epon 812, and sectioned. After staining with uranium acetate and lead citrate, the sections were examined under Hatachy H-600 transmission electron microscope.

\section{Flow cytometry}

After mice were killed, peripheral blood samples were collected in heparin-coated tubes. Peripheral blood $(50 \mu \mathrm{L})$ from each mouse was centrifuged to prepare serum. The remaining peripheral blood was used for peripheral blood leukocyte preparation by Ficoll centrifugation. Spleen was removed and pulverized for splenocyte separation. After red blood cell lysis, washing, and centrifugation, splenocytes were obtained.

Peripheral blood leukocytes and splenocytes were first stained with fluorescein isothiocyanate-conjugated antimouse CD4 and phycoerythrin-conjugated anti-mouse CD25 (eBioscience, San Diego, CA, USA). For Foxp3 staining, cells were treated with allophycocyanin-conjugated antimouse Foxp3 according to the manufacturer's instructions (eBioscience). After staining, cells were analyzed with a FACSCalibur flow cytometer (BD-Bioscience, San Jose, CA, USA), using FlowJo software (Tree Star, San Carlos, CA, USA). The percentage of Tregs in total peripheral blood leukocytes and splenocytes was calculated.

\section{TGF- $\beta$ I, PGE2, and COX2 measurement}

Serum samples from mice were used for the measurement of serum levels of TGF- $\beta 1$, prostaglandin E2 (PGE2), and cyclooxygenase 2 (COX2) by the enzyme-linked immunosorbent assay (ELISA). The serum levels of TGF- $\beta 1$ were measured using a Quantikine mouse TGF- $\beta 1$ ELISA Kit (R\&D Systems, Minneapolis, MN, USA), according to the manufacturer's instructions. ${ }^{27}$ The serum levels of PGE2 and COX2 were measured using a mouse PGE2 ELISA Kit and a mouse COX2 ELISA kit (MyBioSource, San Diego, CA, USA), respectively, according to the manufacturer's instructions.

\section{Statistical analysis}

Quantitative data were expressed as the mean \pm standard deviation. The statistical analysis was carried out using SPSS Statistics 17.0 software (SPSS Inc., Chicago, IL, USA).
Statistical differences between control group without flavonoid administration and other groups were compared using the Wilcoxon rank sum test for $P$-value calculation. All $P$-values lower than 0.05 were considered significant.

\section{Results \\ Effects of total flavonoids on mouse growth}

All groups of mice had similar baseline body weights (Figure 1A). At the end point, the group of mice receiving the LLC cell inoculation but without flavonoid treatment had significantly decreased body weights compared with the control group (Figure 1B), suggesting tumor growth suppressed the increase of mouse body weights. Administration of total flavonoids from Radix Tetrastigmae at higher doses significantly rescued the body weight gain in mice inoculated with LLC cells (Figure 1B). The results indicated that total flavonoids from Radix Tetrastigmae improved the overall health of the mice by suppressing tumor growth in mice.

\section{Effects of total flavonoids on tumor growth}

After LLC cell inoculation, tumor lesions became visible at day 5. After day 7, the administration of total flavonoids significantly inhibited tumor growth in mice at a dose-dependent manner in comparison with the untreated group (Figure 2A). At day 14, the lower-dose group also showed a significant reduction of tumor volumes compared with the untreated group (Figure 2B). The results suggested that total flavonoids from Radix Tetrastigmae suppressed tumor growth in mice.

At day 14, the administration of total flavonoids from Radix Tetrastigmae at a high dose significantly suppressed the increase of tumor weights in mice (Figure 3A and B). Interestingly, the administration of total flavonoids from Radix Tetrastigmae at a low dose at day 14 also significantly inhibited the increase of tumor volumes in mice (Figure 3C). As compared with tumor cells from mice without flavonoid administration (Figure 3D), analysis by transmission electron microscopy indicated that the treatment by a low dose of total flavonoids induced the formation of irregular nucleus and bubble-like structures in the cytoplasm and loss of normal nucleoli (Figure 3E), suggesting that flavonoid administration led to apoptosis of tumor cells in mice. Thus, it appeared that flavonoids from Radix Tetrastigmae suppressed tumor growth in vivo.

\section{Effects of total flavonoids on Treg number}

Because flavonoids from Radix Tetrastigmae could inhibit tumor growth in mice (Figures 2 and 3), and Tregs play a 
A

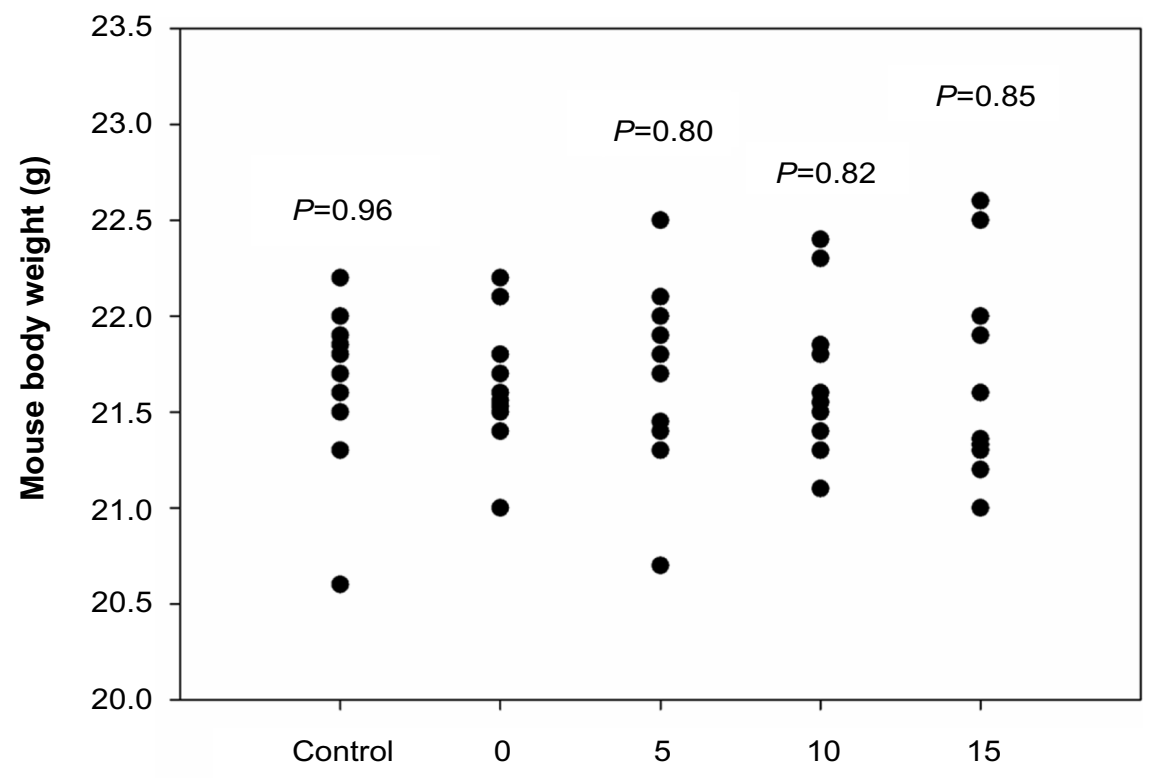

Tumor + flavonoids $(\mathrm{mg} / \mathrm{kg})$

B

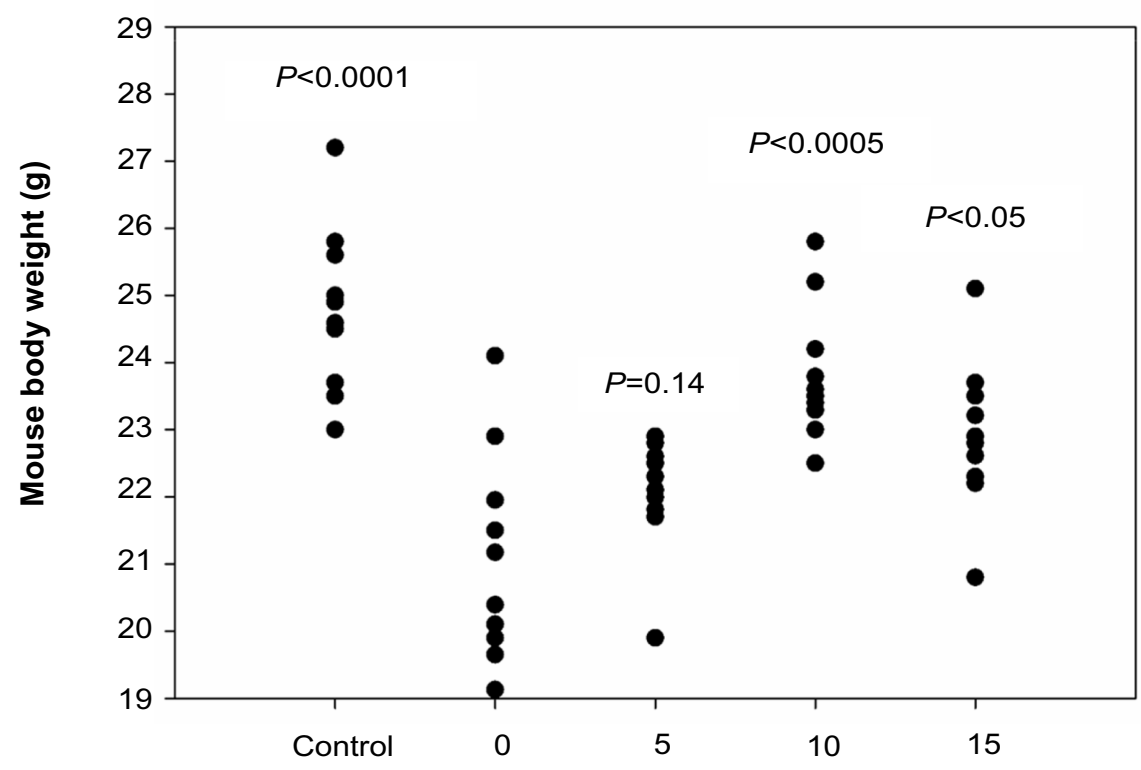

Tumor + flavonoids $(\mathrm{mg} / \mathrm{kg})$

Figure I Effects of flavonoids from Radix Tetrastigmae on mouse body weight.

Notes: The body weights of mice were determined before Lewis lung carcinoma tumor inoculation and flavonoid administration (A) and at day 14 after tumor inoculation and treatment (B). All P-values were calculated from comparison between the tumor inoculation group without flavonoid administration and other groups.

critical role in the suppression of antitumor immunity, ${ }^{12,13,17}$ we aimed to investigate whether flavonoids could inhibit Treg development in mice. Tregs were defined as CD25+ and FoxP3+ CD4+ T cells (Figure 4) and can inhibit the functions of other CD4 T cells and cytotoxic CD8 T cells by means of cell-cell contact or secretion of the inhibitory cytokines interleukin 10 and TGF- $\beta .{ }^{16}$ The results showed that flavonoids from Radix Tetrastigmae significantly reduced Treg number in peripheral blood leukocytes (Figures 4E, F and 5A) and, at higher doses, decreased Treg number in the spleen as well (Figure 5B). The results suggested that flavonoids from Radix Tetrastigmae suppress Treg development, and thus inhibit tumor growth in mice. 
A

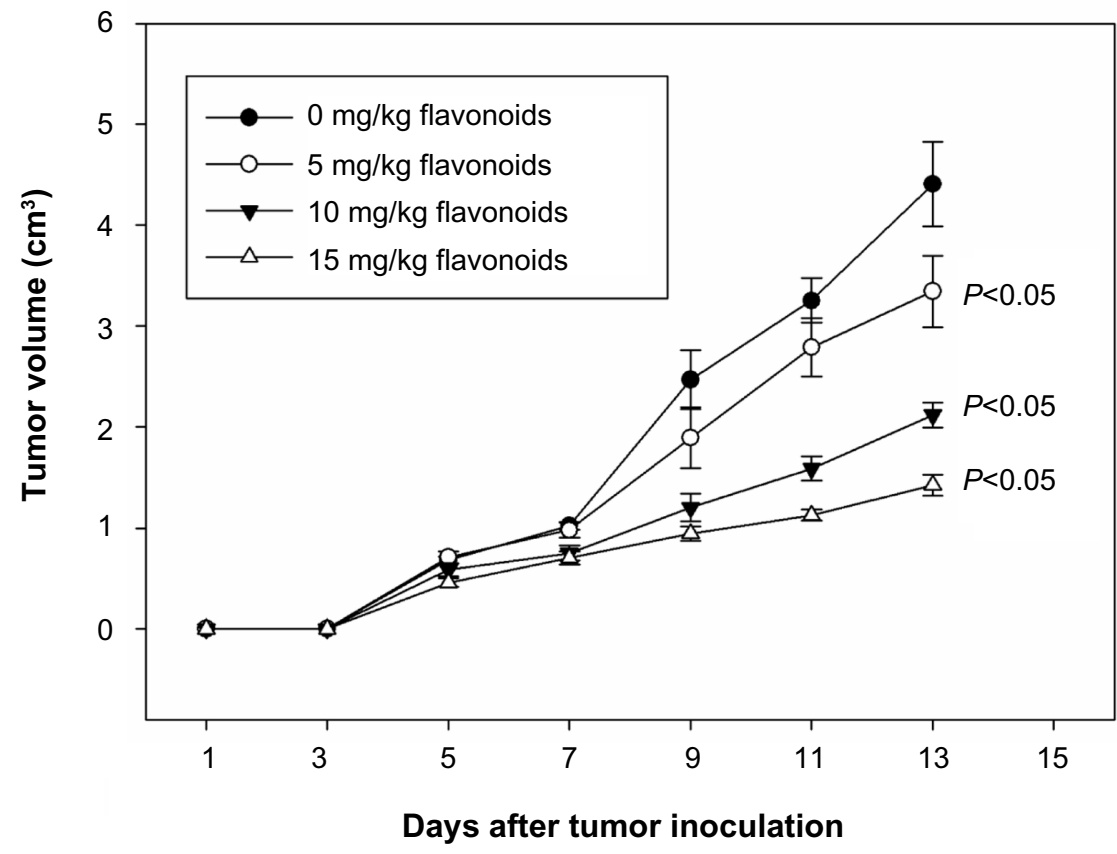

B

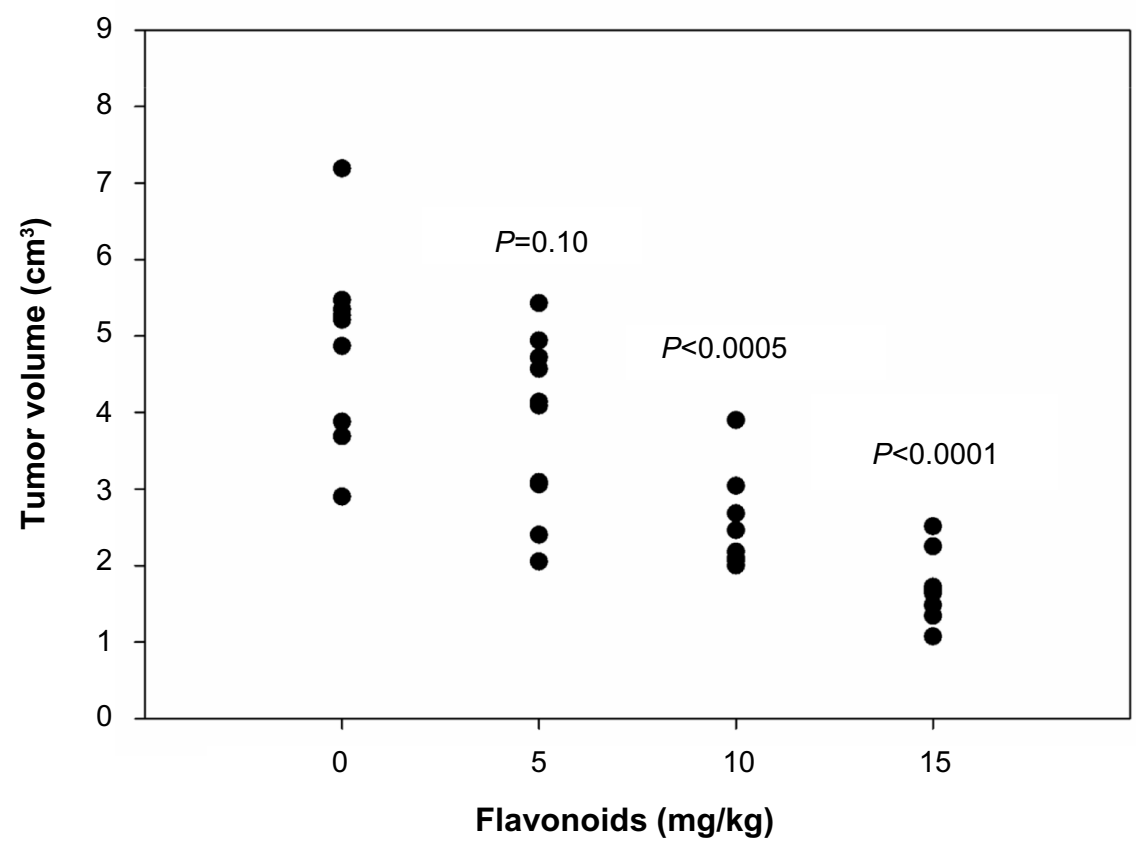

Figure 2 Effects of flavonoids from Radix Tetrastigmae on tumor growth.

Notes: (A) Changes of tumor volumes in mice were monitored daily after Lewis lung carcinoma tumor inoculation and flavonoid administration. (B) Differences of tumor volumes in mice between the tumor inoculation group without flavonoid administration and other groups were compared at day 14 after Lewis lung carcinoma tumor inoculation and flavonoid administration. All P-values were calculated from comparison between the tumor inoculation group without flavonoid administration and other groups.

\section{Effects of total flavonoids on the expression of TGF- $\beta$ I, PGE2, and COX2}

It has been reported that several factors such as TGF- $\beta 1$, PGE2, and COX2 play important roles in the induction, maintenance, and functions of Tregs. ${ }^{28,29}$ Thus, to understand the mechanisms of flavonoid-mediated downregulation of Tregs, we examined the effects of total flavonoids on these factors. ELISA analysis showed that the administration of total flavonoids from Radix Tetrastigmae significantly decreased the serum levels of TGF- $\beta 1$, PGE2, and COX2 in the peripheral blood of mice (Figure 6), suggesting that flavonoids from Radix Tetrastigmae 
A

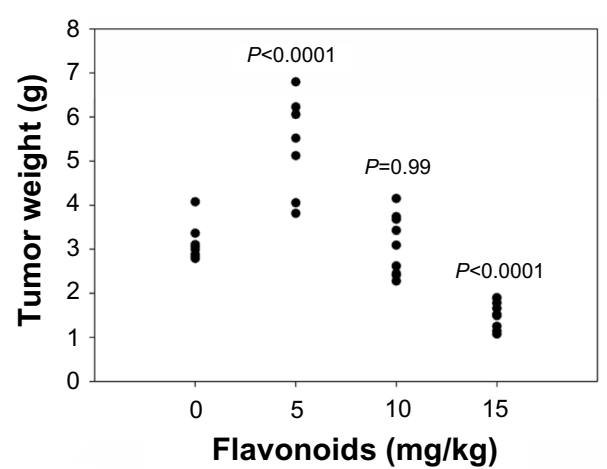

C

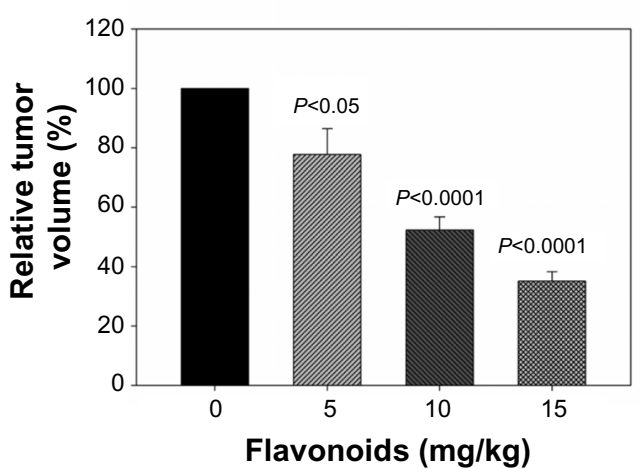

B

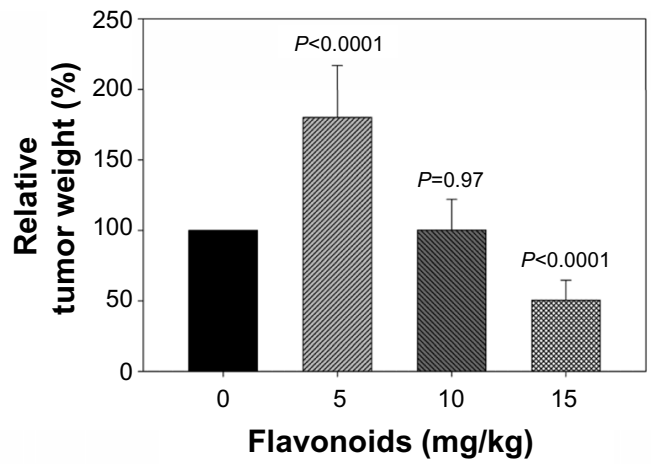

D E

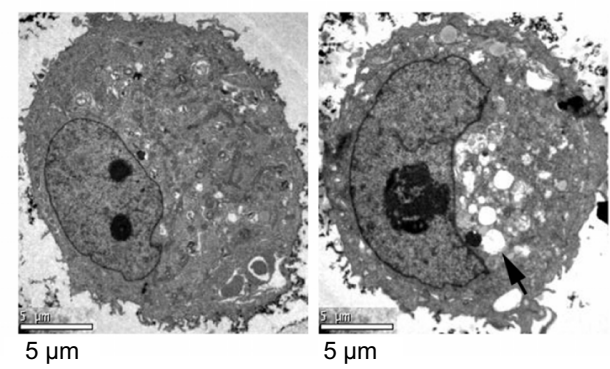

Figure 3 (A) Effects of flavonoids from Radix Tetrastigmae on tumor weight.

Notes: The weights of tumors in mice were measured at day 14 after Lewis lung carcinoma tumor inoculation and flavonoid administration. (B) Inhibitory activities of flavonoids in tumor weight increase are shown. (C) Similar to increases in tumor weight, the inhibitory activities of flavonoids in tumor volume increase were also presented. The cellular structures of tumor cells from mice at day 14 after $5 \mathrm{mg} / \mathrm{kg}$ flavonoid administration were examined by electron microscopy (E), and compared with those from mice without flavonoid administration (D). Arrow bar in (E) indicates the bubble-like structures in tumor cells induced by flavonoid treatment. All $P$-values were calculated from comparison between the tumor inoculation group without flavonoid administration and other groups.

may downregulate the expression of TGF- $\beta 1$, PGE2, and COX2, leading to the suppression of Tregs and inhibition of tumor growth in mice.

\section{Discussion}

It has been shown that flavonoids can induce apoptosis and inhibit proliferation of tumor cells, and thus have antitumor activities. $^{2-4}$ Our previous studies revealed that flavonoids from Radix Tetrastigmae induce apoptosis and inhibit the growth of gastric cancer cells in vitro. ${ }^{23}$ Our current study revealed that administration of flavonoids from Radix Tetrastigmae significantly inhibits tumor growth in a murine cancer model (Figures 2 and 3). Therefore, flavonoids may be used as antitumor agents in the prevention and treatment of cancer. Clinical studies have shown that flavonoids may have great implications in the prevention and treatment of cancer in humans. ${ }^{30-32}$ The results suggest that flavonoids from Radix Tetrastigmae may be good candidates for clinical trials in the prevention and treatment of human cancers.

It is obvious that the administration of total flavonoids from Radix Tetrastigmae at a low dose in mice significantly suppressed the increase of tumor volumes (Figures 2 and 3C) but increased the tumor weights at day 14 after LLC tumor inoculation (Figure 3A and B). Analysis by transmission electron microscopy indicated that treatment by a low dose of total flavonoids stimulated the formation of bubblelike structures in the cytoplasm of tumor cells in mice (Figure $3 \mathrm{E}$ ), suggesting that the administration of flavonoids may induce apoptosis of tumor cells. It is possible that the bubble-like structures in the cytoplasm of tumor cells may be full of fluids, which may be responsible for the increase of tumor weights in mice at day 14 after tumor inoculation and flavonoid administration at a low dose (Figure 3A and B). The results suggested that a higher dose of total flavonoids from Radix Tetrastigmae would be preferred for cancer treatment.

It has been well documented that antitumor immune responses play a pivotal role in cancer development and treatment. ${ }^{33-35}$ Tregs have important functions in the regulation of antitumor immune responses. ${ }^{12,13,36}$ Little is known about the effects of flavonoids on the regulation of antitumor immune responses. It has been reported that wogonin was 
A

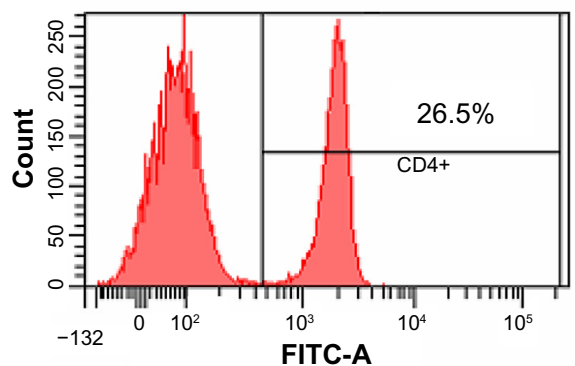

C

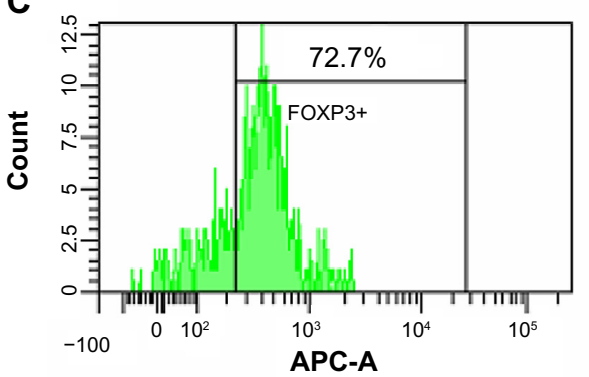

E

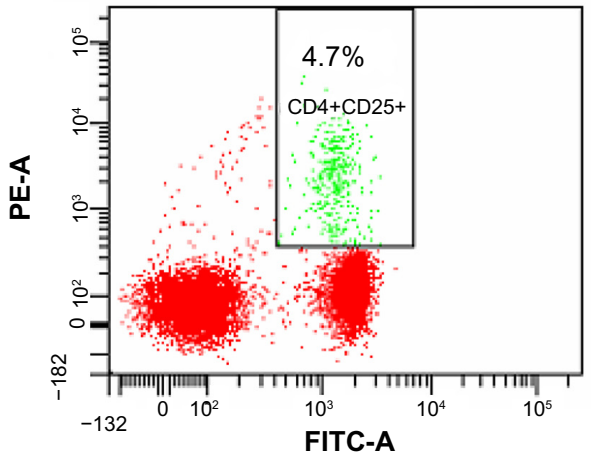

B

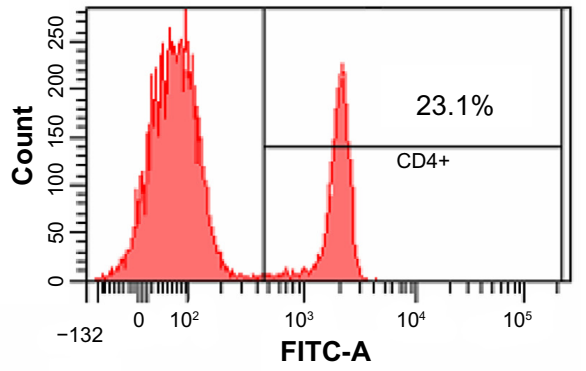

D

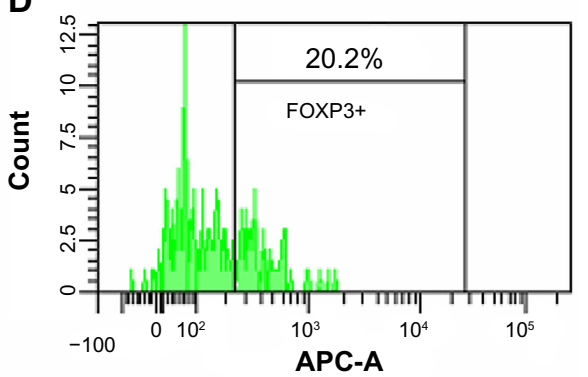

F

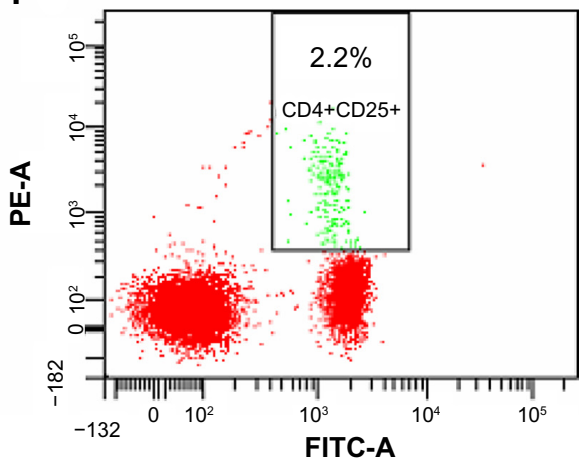

Figure 4 Flow cytometry analysis of regulatory T cells in mice.

Notes: Peripheral blood leukocytes and splenocytes were stained with fluorescein isothiocyanate (FITC)-conjugated anti-mouse CD4 and phycoerythrin-conjugated antimouse CD25. After cell fixation, the cells were treated with allophycocyanin (APC)-conjugated anti-mouse Foxp3. Then, the cells were analyzed by flow cytometry for determination of regulatory T cell quantity. Representative data showing FITC-conjugated CD4-staining cells are presented in peripheral blood leukocytes from mice without flavonoid administration (A) and with $15 \mathrm{mg} / \mathrm{kg}$ flavonoid administration (B) after Lewis lung carcinoma tumor inoculation. Representative data showing allophycocyaninconjugated FoxP3-staining cells are also shown in peripheral blood leukocytes from mice without flavonoid administration (C) and with flavonoid administration (D) and are used in the gating for CD4+CD25+ regulatory T cells in peripheral blood leukocytes from mice without flavonoid administration (E) and with flavonoid administration (F).

the dominant flavonoid in Scutellaria ocmulgee leaf extract and that the oral administration of $S$. ocmulgee leaf extract significantly inhibited intratumoral TGF- $\beta 1$ level and Treg cell frequency in F344 rats transplanted subcutaneously with F98 gliomas. ${ }^{37}$ Thus, we were interested in understanding whether flavonoids from Radix Tetrastigmae could inhibit Treg in LLC mouse model. Our study showed that flavonoids from Radix Tetrastigmae significantly decreased Tregs in mice (Figure 5), suggesting that flavonoids from Radix Tetrastigmae may have a regulatory role in the antitumor immunity by downregulating Tregs. It has been reported that Baicalin, a flavonoid from Huangqin (Scutellaria baicalensis Georgi), promotes Treg development. ${ }^{38}$
Thus, different flavonoids may have opposite effects on Treg development.

TGF- $\beta$ has been shown to play an important role in the induction, maintenance, and function of Tregs. ${ }^{28,39}$ Further studies demonstrated that TGF- $\beta$ induced COX2 expression. ${ }^{40} \mathrm{COX} 2$ regulates PGE2 expression, which was shown to induce FoxP3 expression. ${ }^{41}$ Thus, the COX2-PGE2 pathway is involved in the induction, maintenance, and function of Tregs. ${ }^{29}$ Our analysis revealed that flavonoids from Radix Tetrastigmae significantly decreased the serum levels of TGF- $\beta 1$, PGE2, and COX2 in mice (Figure 6). These results therefore suggest that flavonoids from Radix Tetrastigmae may suppress the expression of TGF- $\beta$, PGE2, and COX2, and thus inhibit Treg development. 
A

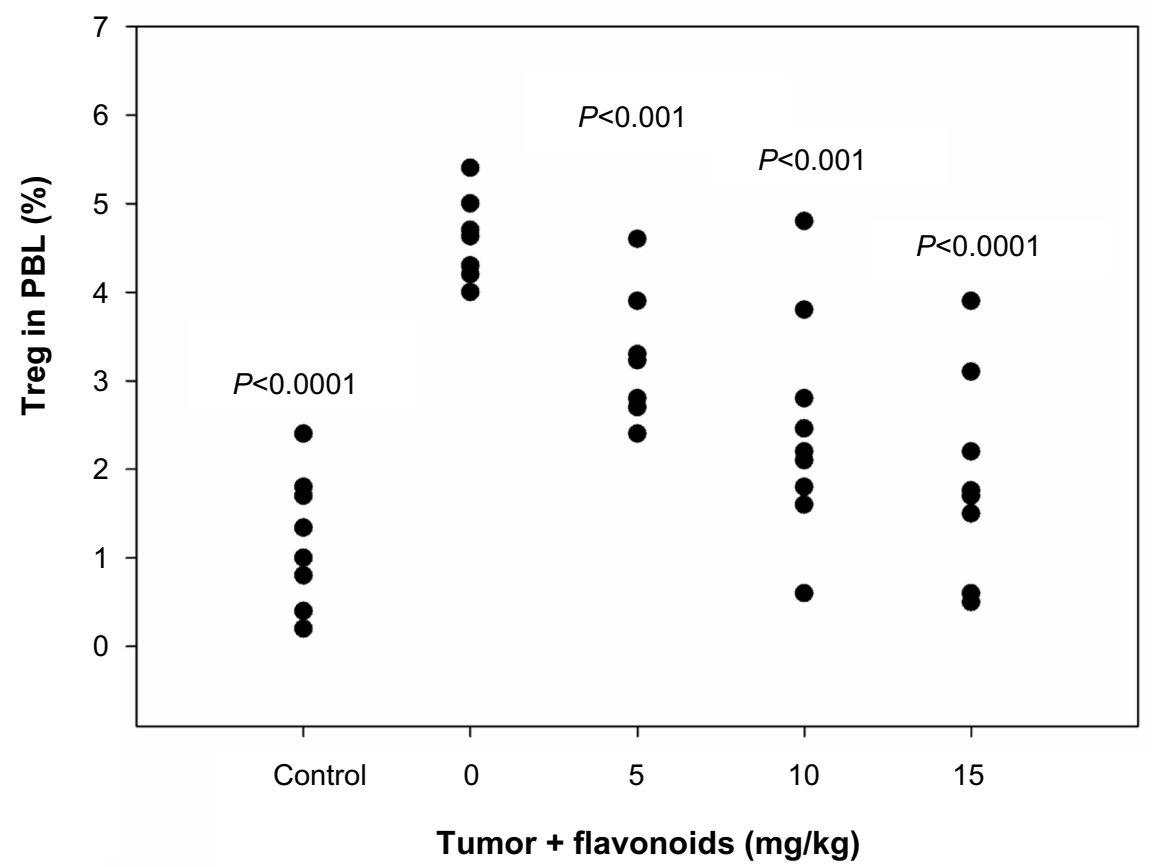

B

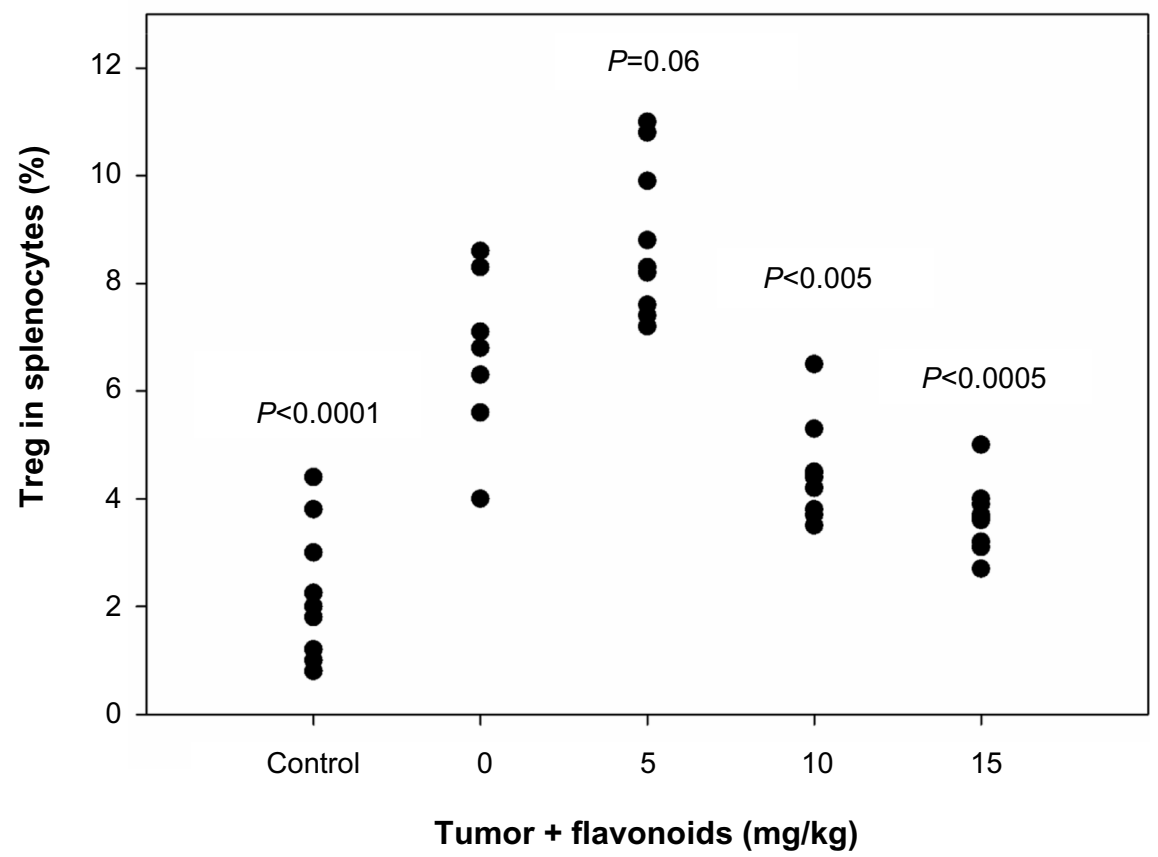

Figure 5 Effects of flavonoids from Radix Tetrastigmae on regulatory T cells in vivo.

Notes: After antibody staining and flow cytometry analysis, the changes of regulatory $\mathrm{T}$ cells in mice at day 14 after Lewis lung carcinoma tumor inoculation and flavonoid administration were determined in peripheral blood leukocytes $(\mathbf{A})$ and splenocytes $(\mathbf{B})$. All $P$-values were calculated from comparison between the tumor inoculation group without flavonoid administration and other groups.

Abbreviations: Treg, regulatory T cells; PBL, peripheral blood leukocytes.

High-performance liquid chromatography analysis showed that quercetin and kaempferol were present in total flavonoids from Radix Tetrastigmae. It has been reported that both quercetin and kaempferol have anticancer function. ${ }^{42-44} \mathrm{~A}$ recent study indicated that quercetin could suppress Treg expansion. ${ }^{45}$
Therefore, it is possible that quercetin in flavonoids from Radix Tetrastigmae may play a major role in the inhibition of Treg and tumor growth. Further studies, however, will be needed to understand the compositions and their functions in total flavonoids from Radix Tetrastigmae for cancer treatment. 
A

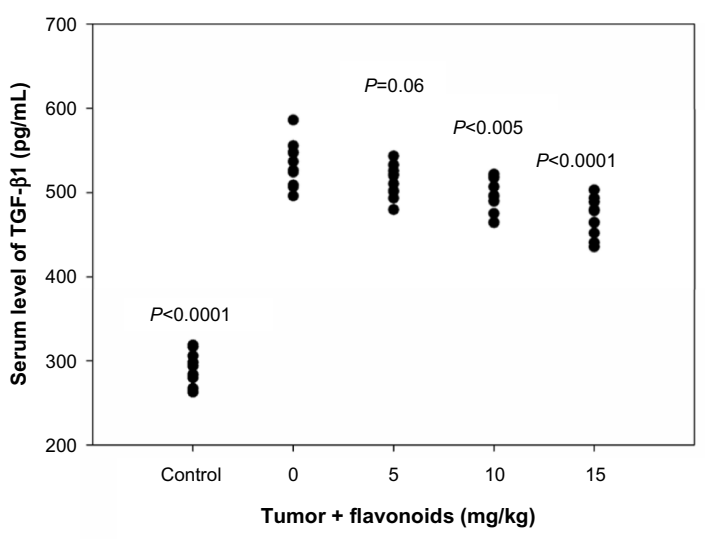

B
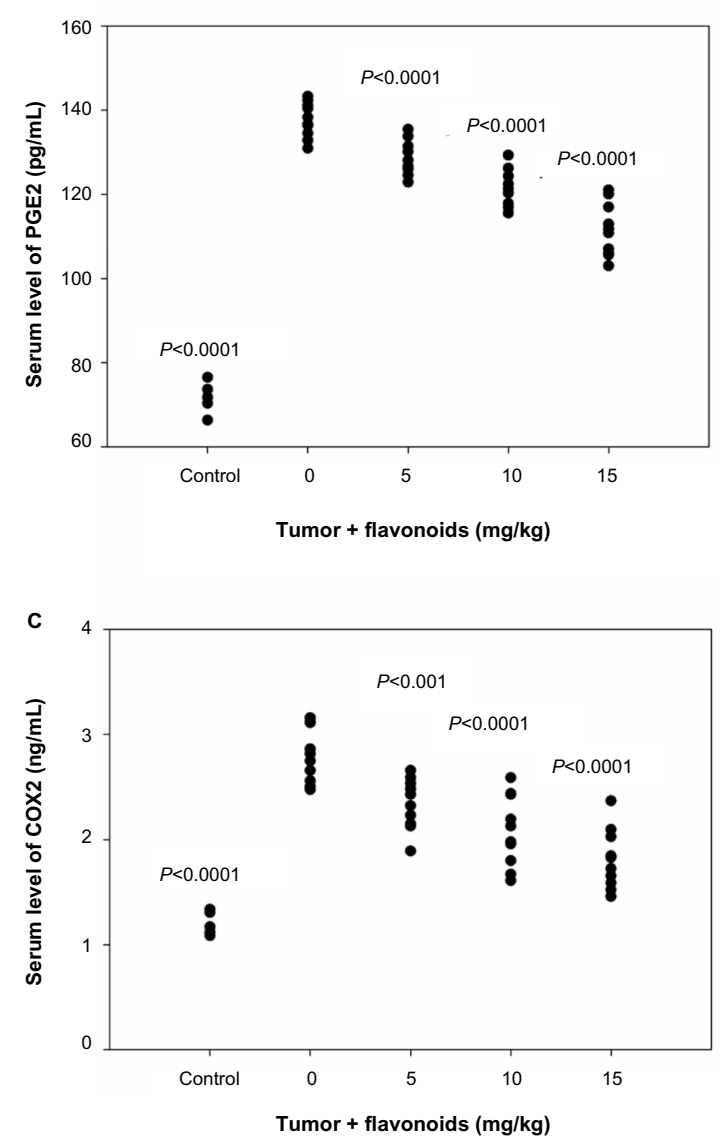

Figure 6 Effects of flavonoids from Radix Tetrastigmae on serum levels of transforming growth factor beta $\beta$ I, prostaglandin E2, and cyclooxygenase 2 .

Notes: Serum samples were prepared from mice at day 14 after Lewis lung carcinoma tumor inoculation and flavonoid administration and used in the determination of serum levels of transforming growth factor beta $\beta \mathrm{I}(\mathbf{A})$, prostaglandin E2 $(\mathbf{B})$, and cyclooxygenase 2 (C) by enzyme-linked immunosorbent assay. All $P$-values were calculated from comparison between the tumor inoculation group without flavonoid administration and other groups.

Abbreviations: TGF- $\beta$ I, transforming growth factor beta $\beta$ I; PGE2, prostaglandin E2; COX2, cyclooxygenase 2 .

\section{Acknowledgments}

This work was supported by a grant (Y12H270055 to ZF) from the Natural Science Foundation of Zhejiang Province, People's Republic of China; a "Taishan Scholar" Special Fund (109, 2012 to JZ) from Shandong Government,
People's Republic of China; and a grant from National Institute of Health (R01HL116626 to DF).

\section{Disclosure}

The authors report no conflicts of interest in this work.

\section{References}

1. Babu BV, Konduru NK, Nakanishi W, Hayashi S, Ahmed N, Mitrasinovic PM. Experimental and theoretical advances in functional understanding of flavonoids as anti-tumor agents. Anticancer Agents Med Chem. 2013;13(2):307-332.

2. Wei A, Wu G, Xiong C, Zhou D, Cai Y, Ruan J. [Flavonoids with special B-ring from Macrothelypteris viridifrons and their anti-proliferative effects on tumor cell]. Zhongguo Zhong Yao Za Zhi. 2011;36(5): 582-584. Chinese.

3. Kim YA, Kim H, Seo Y. Antiproliferative effect of flavonoids from the halophyte Vitex rotundifolia on human cancer cells. Nat Prod Commun. 2013;8(10):1405-1408.

4. Szliszka E, Czuba ZP, Jernas K, Król W. Dietary flavonoids sensitize HeLa cells to tumor necrosis factor-related apoptosis-inducing ligand (TRAIL). Int J Mol Sci. 2008;9(1):56-64.

5. Han J, Sun M, Cui Y, et al. Kushen flavonoids induce apoptosis in tumor cells by inhibition of NF-kappaB activation and multiple receptor tyrosine kinase activities. Phytother Res. 2007;21(3): 262-268.

6. Schindler R, Mentlein R. Flavonoids and vitamin E reduce the release of the angiogenic peptide vascular endothelial growth factor from human tumor cells. J Nutr. 2006;136(6):1477-1482.

7. Piantelli M, Rossi C, Iezzi M, et al. Flavonoids inhibit melanoma lung metastasis by impairing tumor cells endothelium interactions. J Cell Physiol. 2006;207(1):23-29.

8. Lotito SB, Frei B. Dietary flavonoids attenuate tumor necrosis factor alpha-induced adhesion molecule expression in human aortic endothelial cells. Structure-function relationships and activity after first pass metabolism. J Biol Chem. 2006;281(48):37102-37110.

9. Yao H, Xu W, Shi X, Zhang Z. Dietary flavonoids as cancer prevention agents. J Environ Sci Health C Environ Carcinog Ecotoxicol Rev. 2011;29(1):1-31.

10. Russo P, Del Bufalo A, Cesario A. Flavonoids acting on DNA topoisomerases: recent advances and future perspectives in cancer therapy. Curr Med Chem. 2011;9(31):5287-5293.

11. Romagnolo DF, Selmin OI. Flavonoids and cancer prevention: a review of the evidence. J Nutr Gerontol Geriatr. 2012;31(3):206-238.

12. Banerjee A, Vasanthakumar A, Grigoriadis G. Modulating $\mathrm{T}$ regulatory cells in cancer: how close are we? Immunol Cell Biol. 2013;91(5):340-349.

13. Galgani M, Di Giacomo A, Matarese G, La Cava A. The Yin and Yang of CD4(+) regulatory T cells in autoimmunity and cancer. Curr Med Chem. 2009;16(35):4626-4631.

14. Hori S, Nomura T, Sakaguchi S. Control of regulatory T cell development by the transcription factor Foxp3. Science. 2003;299(5609): 1057-1061.

15. Dranoff G. The therapeutic implications of intratumoral regulatory T cells. Clin Cancer Res. 2005;11(23):8226-8229.

16. Watanabe MA, Oda JM, Amarante MK, Cesar Voltarelli J. Regulatory T cells and breast cancer: implications for immunopathogenesis. Cancer Metastasis Rev. 2010;29(4):569-579.

17. Oleinika K, Nibbs RJ, Graham GJ, Fraser AR. Suppression, subversion and escape: the role of regulatory $\mathrm{T}$ cells in cancer progression. Clin Exp Immunol. 2013;171(1):36-45.

18. Ménétrier-Caux C, Curiel T, Faget J, Manuel M, Caux C, Zou W. Targeting regulatory T cells. Target Oncol. 2012;7(1):15-28.

19. Ghiringhelli F, Puig PE, Roux S, et al. Tumor cells convert immature myeloid dendritic cells into TGF-beta-secreting cells inducing CD4+CD25+ regulatory T cell proliferation. J Exp Med. 2005;202(7): 919-929. 
20. Zou W. Regulatory T cells, tumour immunity and immunotherapy. Nat Rev Immunol. 2006;6(4):295-307.

21. Ghiringhelli F, Menard C, Puig PE, et al. Metronomic cyclophosphamide regimen selectively depletes $\mathrm{CD} 4+\mathrm{CD} 25+$ regulatory $\mathrm{T}$ cells and restores $\mathrm{T}$ and $\mathrm{NK}$ effector functions in end stage cancer patients. Cancer Immunol Immunother. 2007;56(5):641-648.

22. Zhang L, Dermawan K, Jin M, et al. Differential impairment of regulatory $\mathrm{T}$ cells rather than effector $\mathrm{T}$ cells by paclitaxel-based chemotherapy. Clin Immunol. 2008;129(2):219-229.

23. Feng Z-Q, Ni K-F, He Y, et al. [Experimental study on effect of Tetrastigma hemsleyanum Diels et Gilg flavone on inducing apoptosis of SGC-7901 cell line in vitro.] Chin J Clin Pharmacol Ther. 2006;11: 669-672. Chinese.

24. Liu B, Yang J, Ma Y, Yuan E, Chen C. Antioxidant and angiotensin converting enzyme (ACE) inhibitory activities of ethanol extract and pure flavonoids from Adinandra nitida leaves. Pharm Biol. 2010;48(12):1432-1438.

25. Chen L, Ding L, Yu A, et al. Continuous determination of total flavonoids in Platycladus orientalis (L.) Franco by dynamic microwave-assisted extraction coupled with on-line derivatization and ultraviolet-visible detection. Anal Chim Acta. 2007;596(1):164-170.

26. Euhus DM, Hudd C, LaRegina MC, Johnson FE. Tumor measurement in the nude mouse. J Surg Oncol. 1986;31(4):229-234.

27. Sriarj W, Aoki K, Ohya K, Takahashi M, Takagi Y, Shimokawa H. TGF- $\beta$ in dentin matrix extract induces osteoclastogenesis in vitro. Odontology. Epub December 24, 2013.

28. Horwitz DA, Zheng SG, Gray JD. Natural and TGF-beta-induced Foxp3(+)CD4(+) CD25(+) regulatory $\mathrm{T}$ cells are not mirror images of each other. Trends Immunol. 2008;29(9):429-435.

29. Sharma S, Yang SC, Zhu L, et al. Tumor cyclooxygenase-2/ prostaglandin E2-dependent promotion of FOXP3 expression and CD4+ CD25+ T regulatory cell activities in lung cancer. Cancer Res. 2005;65(12):5211-5220.

30. Knekt P, Järvinen R, Seppänen R, et al. Dietary flavonoids and the risk of lung cancer and other malignant neoplasms. Am J Epidemiol. 1997;146(3):223-230.

31. Le Marchand L. Cancer preventive effects of flavonoids - a review. Biomed Pharmacother. 2002;56(6):296-301.

32. Ferry DR, Smith A, Malkhandi J, et al. Phase I clinical trial of the flavonoid quercetin: pharmacokinetics and evidence for in vivo tyrosine kinase inhibition. Clin Cancer Res. 1996;2(4):659-668.
33. Jinushi $\mathrm{M}$. The role of innate immune signals in antitumor immunity. Oncoimmunology. 2012;1(2):189-194.

34. Ostrand-Rosenberg S. Immune surveillance: a balance between protumor and antitumor immunity. Curr Opin Genet Dev. 2008;18(1):11-18.

35. Hinrichs CS, Rosenberg SA. Exploiting the curative potential of adoptive T-cell therapy for cancer. Immunol Rev. 2014;257(1):56-71.

36. Kadagidze ZG, Chertkova AI, Slavina EG. [Regulatory T-cells and their role in antitumor immune response]. Vopr Onkol. 2009;55(3): 269-277. Russian.

37. Dandawate S, Williams L, Joshee N, et al. Scutellaria extract and wogonin inhibit tumor-mediated induction of T(reg) cells via inhibition of TGF- $\beta 1$ activity. Cancer Immunol Immunother. 2012;61(5): 701-711.

38. Yong X, Xiao YF, Luo G, et al. Strategies for enhancing vaccineinduced CTL antitumor immune responses. J Biomed Biotechnol. 2012;2012:605045.

39. Ogawa C, Tone Y, Tsuda M, Peter C, Waldmann H, Tone M. TGF- $\beta$ mediated Foxp3 gene expression is cooperatively regulated by Stat 5 , Creb, and AP-1 through CNS2. J Immunol. 2014;192(1):475-483.

40. Neil JR, Johnson KM, Nemenoff RA, Schiemann WP. Cox-2 inactivates Smad signaling and enhances EMT stimulated by TGF-beta through a PGE2-dependent mechanisms. Carcinogenesis. 2008;29(11):2227-2235.

41. Baratelli F, Lin Y, Zhu L, et al. Prostaglandin E2 induces FOXP3 gene expression and $\mathrm{T}$ regulatory cell function in human $\mathrm{CD} 4+\mathrm{T}$ cells. J Immunol. 2005;175(3):1483-1490.

42. Camargo CA, da Silva ME, da Silva RA, Justo GZ, Gomes-Marcondes MC, Aoyama $\mathrm{H}$. Inhibition of tumor growth by quercetin with increase of survival and prevention of cachexia in Walker 256 tumor-bearing rats. Biochem Biophys Res Commun. 2011;406(4):638-642.

43. Hirpara KV, Aggarwal P, Mukherjee AJ, Joshi N, Burman AC. Quercetin and its derivatives: synthesis, pharmacological uses with special emphasis on anti-tumor properties and prodrug with enhanced bio-availability. Anticancer Agents Med Chem. 2009;9(2):138-161.

44. Xie F, Su M, Qiu W, et al. Kaempferol promotes apoptosis in human bladder cancer cells by inducing the tumor suppressor, PTEN. Int J Mol Sci. 2013;14(11):21215-21226.

45. Kim MG, Jung Cho E, Won Lee J, et al. The heat-shock protein-70induced renoprotective effect is partially mediated by CD4+ CD25+ Foxp3 + regulatory T cells in ischemia/reperfusion-induced acute kidney injury. Kidney Int. 2014;85(1):62-71.
OncoTargets and Therapy

\section{Publish your work in this journal}

OncoTargets and Therapy is an international, peer-reviewed, open access journal focusing on the pathological basis of all cancers, potential targets for therapy and treatment protocols employed to improve the management of cancer patients. The journal also focuses on the impact of management programs and new therapeutic agents and protocols on

\section{Dovepress}

patient perspectives such as quality of life, adherence and satisfaction The manuscript management system is completely online and includes a very quick and fair peer-review system, which is all easy to use. Visit http://www.dovepress.com/testimonials.php to read real quotes from published authors. 